12-16September 2016 Ljubljana,Slovenia

\title{
New Frontiers in Black Hole Astrophysics
}

Edited by

\section{Andreja Gomboc}

ISSN 1743-9213

International Astronomical Union

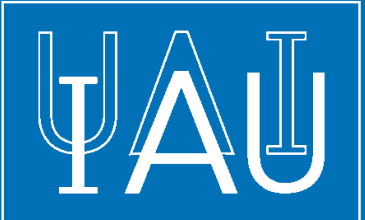

CAMBRIDGE

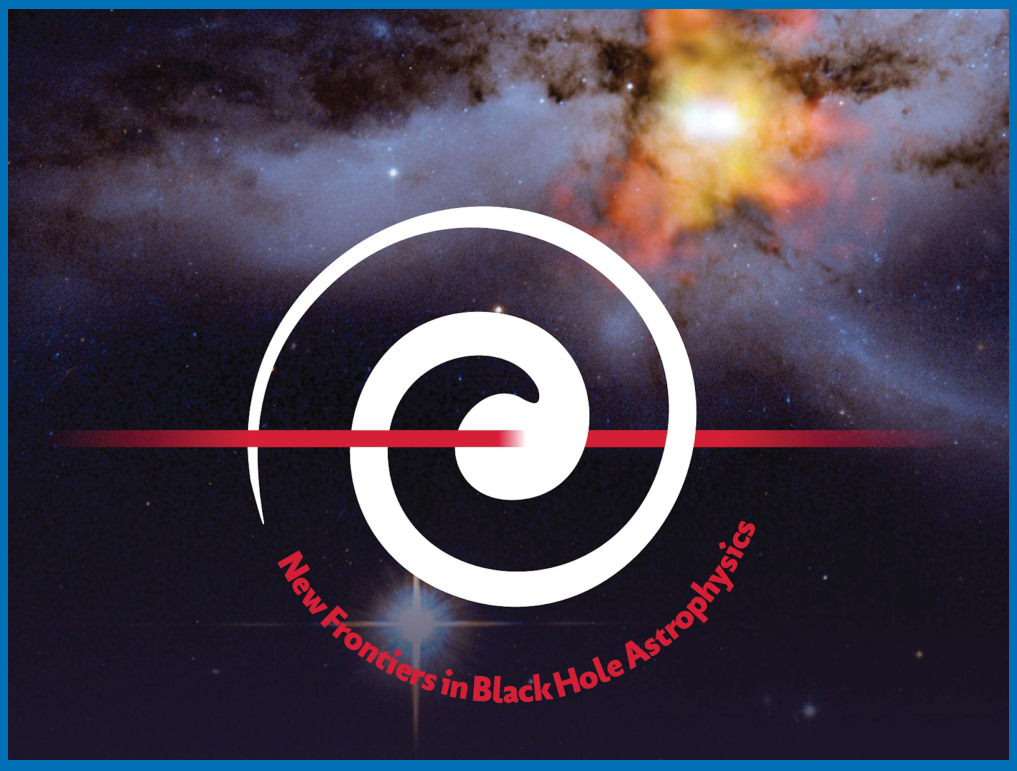


NEW FRONTIERS IN BLACK HOLE ASTROPHYSICS

IAU SYMPOSIUM 324

COVER ILLUSTRATION:

Logo of the IAU Symposium 324 on the background of NGC 6240 (Logo Credit: Branko Žalar, Photo Credits: X-ray: NASA/CXC/MIT/C. Canizares, M. Nowak; Optical: NASA/STScI) 
IAU SYMPOSIUM PROCEEDINGS SERIES

\author{
Chief Editor \\ PIERO BENVENUTI, IAU General Secretary \\ IAU-UAI Secretariat \\ 98-bis Blvd Arago \\ F-75014 Paris \\ France \\ iau-general.secretary@iap.fr
}

Editor

MARIA TERESA LAGO, IAU Assistant General Secretary

Universidade do Porto

Centro de Astrofísica

Rua das Estrelas

4150-762 Porto

Portugal

mtlago@astro.up.pt 
INTERNATIONAL ASTRONOMICAL UNION

UNION ASTRONOMIQUE INTERNATIONALE

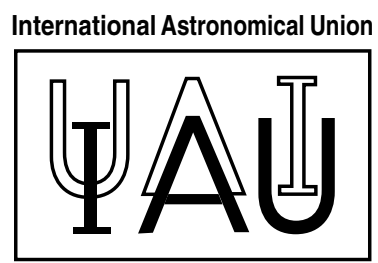

\title{
NEW FRONTIERS IN BLACK HOLE ASTROPHYSICS
}

\author{
PROCEEDINGS OF THE 324th SYMPOSIUM \\ OF THE INTERNATIONAL ASTRONOMICAL \\ UNION HELD IN LJUBLJANA, SLOVENIA \\ SEPTEMBER 12-16, 2016
}

Edited by

ANDREJA GOMBOC

University of Nova Gorica, Slovenia 
CAMBRIDGE UNIVERSITY PRESS

University Printing House, Cambridge CB2 8BS, United Kingdom

1 Liberty Plaza, Floor 20, New York, NY 10006, USA

10 Stamford Road, Oakleigh, Melbourne 3166, Australia

(C) International Astronomical Union 2017

This book is in copyright. Subject to statutory exception and to the provisions of relevant collective licensing agreements, no reproduction of any part may take place without the written permission of the International Astronomical Union.

First published 2017

Printed in the UK by Bell \& Bain, Glasgow, UK

Typeset in System $\mathrm{HT}_{\mathrm{EX}} 2_{\varepsilon}$

A catalogue record for this book is available from the British Library Library of Congress Cataloguing in Publication data

This journal issue has been printed on $\mathrm{FSC}^{\mathrm{TM}}$-certified paper and cover board. FSC is an independent, non-governmental, not-for-profit organization established to promote the responsible management of the world's forests. Please see www.fsc.org for information.

ISBN 9781107169944 hardback

ISSN 1743-9213 


\section{Table of Contents}

Preface $\ldots \ldots \ldots \ldots \ldots \ldots \ldots \ldots \ldots \ldots \ldots \ldots \ldots \ldots \ldots \ldots \ldots \ldots \ldots \ldots \ldots \ldots \ldots \ldots$

Acknowledgements .......................... xiii

Scientific Organizing Committee . . . . . . . $\ldots \ldots \ldots \ldots \ldots \ldots \ldots \ldots \ldots \ldots \ldots$

Conference Photograph $\ldots \ldots \ldots \ldots \ldots \ldots \ldots \ldots \ldots \ldots \ldots \ldots \ldots \ldots \ldots \ldots \ldots$

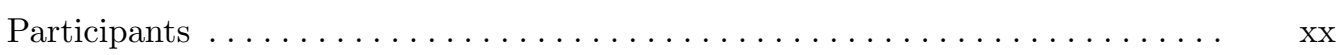

\section{Introduction}

Old/Past/Ancient/Historic Frontiers in Black Hole Astrophysics . . . . . . . . . . V. Trimble

\section{Topic 1. Similarity and Diversity of Black Hole Systems}

Similarity and diversity of black holes - view from the Very High Energies . .... E. Lindfors

Where is the electric current driven in the Blandford-Znajek process? . . . . . . . K. Toma, F. Takahara

Transonic structure of slowly rotating accretion flows with shocks around black

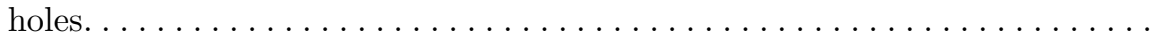

P. Suková, S. Charzyński \&̇ A. Janiuk

Stellar progenitors of black holes: insights from optical and infrared observations I. F. Mirabel

Investigating ultraluminous X-ray sources through their multiwavelength variabil-

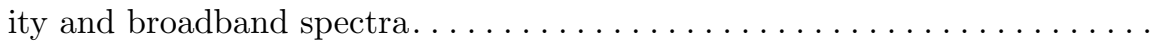

L. Zampieri, E. Ambrosi \& A. Nayerhoda

Black Hole Binaries in Quiescence $\ldots \ldots \ldots \ldots \ldots \ldots \ldots \ldots \ldots \ldots \ldots \ldots \ldots \ldots \ldots \ldots \ldots$

C. D. Bailyn

The search for isolated $\mathrm{BH}$ candidates based on kinematics of pulsars - their former companions in disrupted binaries $\ldots \ldots \ldots \ldots \ldots \ldots \ldots \ldots \ldots \ldots \ldots \ldots \ldots \ldots \ldots$

E. Chmyreva, G. Beskin, V. Dyachenko \& S. Karpov

Prospects for the Discovery of Black Hole Binaries without Mass Accretion with

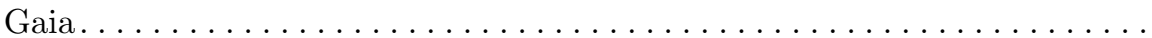

N. Kawanaka, M. Yamaguchi, T. Piran \& T. Bulik

Two optical emission components with different variability in V404 Cygni ..... .

Y. Tachibana, T. Yoshii \& N. Kawai

A pseudo-Newtonian description of any stationary space-time

V. Witzany \& C. Lämmerzahl

\section{Topic 2: Gamma Ray Bursts}

Gamma-ray Bursts Progress and Problems

N. R. Tanvir 
Gamma-Ray Bursts Polarization $\ldots \ldots \ldots \ldots \ldots \ldots \ldots \ldots \ldots \ldots \ldots \ldots \ldots \ldots \ldots \ldots$

D. Götz \& S. Covino

Generation and decay of the magnetic field in collisionless shocks . . . . . . .

M. Garasev \& E. Derishev

Low- $\Gamma$ jets from Compact Binary Mergers as Candidate Electromagnetic Counterparts to Gravitational Wave Sources ......................

G. P. Lamb \& S. Kobayashi

Search for High Energy emission from GRBs with MAGIC . . . . . . . . .

A. Berti for the MAGIC GRB group

Extremely Bright GRB160625B with Short-Soft Precursor and long-hard extended emission: Hints for long-term evolution of the GRB Ejecta . . . . . . . . S.-Q Zhong, J. Lü, H.-J. Lü, H.-M. Zhang, X.-L. Huang \& E.-W. Liang

Lorentz Factor Evolution Patterns within Relativistic Jets of GRBs and AGNs . H.-M. Zhang, D.-B. Lin, T.-T. Lin, B.-R. Liu, X.-L. Huang, S.-Q. Zhong, R.-J. Lu \& E.-W. Liang

Doppler Boosting Effect on the Jet Radiation of Gamma-Ray Bursts and Active Galactic Nuclei . . . . . . . . . . . . . . . . . . . . . . . . . X.-L. Huang, H.-M. Zhang, S.-Q. Zhong \& E.-W. Liang

Untriggered search for rapid optical transients with Mini-MegaTORTORA wide-

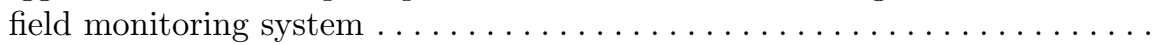

S. Karpov, G. Beskin, A. Biryukov, S. Bondar, E. Ivanov, E. Katkova, N. Orekhova, A. Perkov \& V. Sasyuk

Synergetic Growth of the Rayleigh-Taylor and Richtmyer-Meshkov Instabilities in the Relativistic Jet...........................

J. Matsumoto \& Y. Masada

Spatial Distribution of the Gamma-ray Bursts and the Cosmological Principle. . A. Mészáros

\section{Topic 3: Tidal Disruption Events}

Observational Progress in Identifying and Characterizing Tidal Disruption Flares S. B. Cenko

Relativistic loss cone dynamics: Infall and inspiral rates and branching ratios. . T. Alexander

The role of electromagnetism in tidal disruption events . . . . . . . . . 107 A. $\check{C} a d e \check{z}$

Star Formation Close to Sgr A* and Beyond the Nuclear Cluster...........

F. Yusef-Zadeh \& M. Wardle

What can Fermi LAT observation of the Galactic Centre tell us about its active

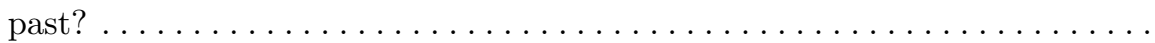

G. Zaharijas, J. Petrović \&S P. Serpico 
Swift J1644+5734: the EVN view. . . . . . . . . . . . . . . .

Z. Paragi, J. Yang, S. Komossa, A. van der Horst, L. I. Gurvits,

R. M. Campbell, D. Giannios \& T. An

Tidal disruption events seen in the XMM-Newton slew survey . . . . . . . .

R. Saxton, S. Komossa, A. Read, P. Lira, K. D. Alexander \& I. Steele

Tidal Disruption Events and stellar-mass black holes in OGLE and Gaia surveys

E. Wyrzykowski, A. Hamanowicz \& K. A. Rybicki

Tidal disruption events from different kinds of astrophysical objects: a preliminary analysis

A. Clerici \& A. Gomboc

Accretion and wind dynamics in tidal disruption events $\ldots \ldots \ldots \ldots \ldots \ldots$

T. Mageshwaran \& A. Mangalam

Explosive nucleosynthesis in tidal disruption events of massive white dwarfs, and

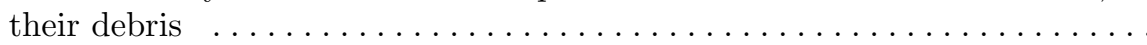

A. Tanikawa, Y. Sato, K. Nomoto, K. Maeda, N. Nakasato \& I. Hachisu

\section{Topic 4: Active Galactic Nuclei}

The remarkable AGN jets . . . . . . . . . . . . . . . . . . . .

S. Komissarov

Observational View of Magnetic Fields in Active Galactic Nuclei Jets . . . . . . . . T. Hovatta

Ultrafast VHE Gamma-Ray Flares of IC $310 \ldots \ldots \ldots \ldots \ldots \ldots \ldots \ldots$

M. V. Barkov, F. Aharonian \& D. V. Khangulyan

Monitoring of the radio galaxy M 87 at Very High Energy with MAGIC during a low emission state between 2012 and $2015 \ldots \ldots \ldots \ldots \ldots \ldots \ldots \ldots$

C. Arcaro, P. Bangale, M. Manganaro, D. Mazin, P. Colin, Ie. Vovk, K. Mannheim for the MAGIC Collaboration, K. Hada, H. E. Jermak, J. P. Madrid, F. Massaro, S. Richter, F. Spanier \& R. C. Walker

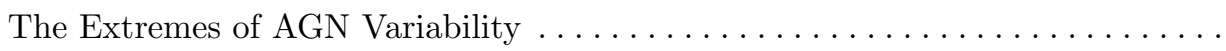
168

S. Komossa, D. Grupe, N. Schartel, L. Gallo, J. L. Gomez, W. Kollatschny, G. Kriss, K. Leighly, A. L. Longinotti, M. Parker, M. Santos-Lleo, D. Wilkins \& $M$. Zetzl

X-ray fluctuation timescale and Black Hole mass relation in AGN . . . . . . . . 172 A. Wandel \& M. Malkan

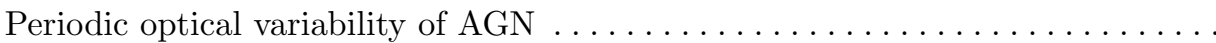
E. Bon, P. Marziani \& N. Bon

Quasi-Periodicities at Year Time Scales in Blazars. . . . . . . . . . . . . 180 S. Covino, A. Sandrinelli \& A. Treves

Radio and $\gamma$-ray loud narrow-line Seyfert 1 galaxies in the spotlight . . . . . . 184 V. Karamanavis, E. Angelakis, S. Komossa, I. Myserlis, D. Blinov $\&$ J. A. Zensus 
The jet detection in radio-loud narrow-line Seyfert 1 galaxies . . . . . . . . . . M. Gu

Elusive Accretion Discs in Low Luminosity AGN . . . . . . . . . . . . .

J.A. Fernández-Ontiveros, M.A. Prieto Escudero, S. Markoff, L. Reb,

D. Espada \& O. González-Martín

A thin disk model for the high efficiency jet in powerful lobe-dominated FRII radio

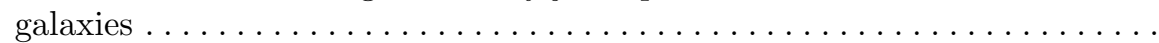

S.-L. Li

Particle-in-cell Simulations of Global Relativistic Jets with Helical Magnetic Fields

I. Duţan, K.-I. Nishikawa, Y. Mizuno, J. Niemiec, O. Kobzar, M. Pohl,

J. L. Gómez, A. Pe'er, J. T. Frederiksen, A. Nordlund, A. Meli, H. Sol,

P. E. Hardee \& D. H. Hartmann

Multi-wavelength flares and magnetic field in blazars: a case study of IBL S5

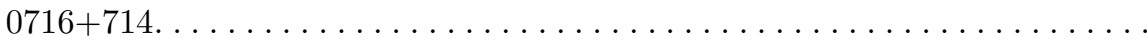

S. Chandra, K. P. Singh \& K. S. Baliyan

Producing ultra high energy cosmic rays from AGN magnetic luminosity . . . . .

C. H. Coimbra-Araújo \& R. C. Anjos

The Energetic Particle Population in Centaurus A...............

P. Chadwick, A. M. Brown, C. Boehm, J. Graham, T. Lacroix EJ J. Silk

Space Telescope and Optical Reverberation Mapping Project: A Leap Forward in

Reverberation Mapping ........................

B. M. Peterson

Studying the outskirts of reverberation mapped AGNs $\ldots \ldots \ldots \ldots \ldots \ldots$

S. Kaspi

Searching for a pair of accreting supermassive black holes in J1425+3231 . . . . .

K. É. Gabányi, S. Frey, Z. Paragi, T. An \& S. Komossa

A Statistical Method for Detecting Gravitational Recoils of Supermassive Black

Holes in Active Galactic Nuclei. . . . . . . . . . . . . . . . . . . . .

P. Raffai, B. Bécsy, Z. Haiman \& Z. Frei

Simulation of AGN feedback and its impact on galaxies $\ldots \ldots \ldots \ldots \ldots \ldots$

M. A. Bourne

MAGIC detection of sub-TEV emission from gravitationally lensed blazar QSO

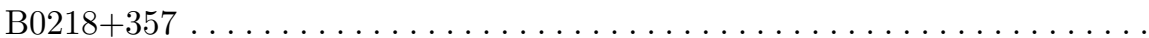

D. Dominis Prester, J. Sitarek, J. Becerra, S. Buson, E. Lindfors, M. Manganaro, D. Mazin, M. Nievas Rosillo, K. Nilsson, A. Stamerra, F. Tavecchio, I. Vovk for the MAGIC Coll. and the Fermi-LAT Coll.

The effect of AGN feedback on Sunyaev-Zeldovich properties of simulated galaxy

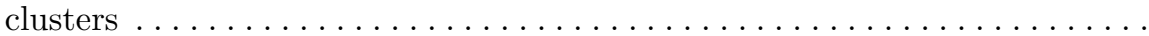

D. Fabjan, S. Planelles, S. Borgani, G. Murante, E. Rasia, V. Biff,

N. Truong, C. Ragone-Figueroa, G. L. Granato, K. Dolag, E. Pierpaoli,

A. M. Beck, L. K. Steinborn \& M. Gaspari 
Empirical multi-wavelength prediction method for Very High Energy Gamma-ray

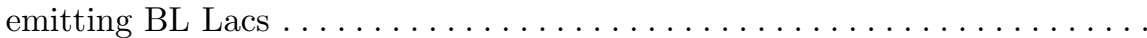

V. Fallah Ramazani, E. Lindfors $\&$ K. Nilsson

Observations of possible jet formation in the binary blazar OJ287 . . . . . . .

H. Jermak, I. A. Steele, G. P. Lamb, M. Valtonen \& S. Zola

Optical variability patterns of radio-quiet and radio-loud quasars. . . . . . . . .

P. Marziani, E. Bon, A. Grieco, N. Bon, D. Dultzin, A. Del Olmo EG

M. D'Onofrio

Highly accreting quasars: a tool for cosmology? . . . . . . . . . . . .

P. Marziani, C. A. Negrete, D. Dultzin, M. L. Martinez-Aldama,

A. Del Olmo, D. Esparza, J. W. Sulentic, M. D'Onofrio, G. M. Stirpe,

E. Bon \& N. Bon

Open our eyes to wider fields in VLBI surveys $\ldots \ldots \ldots \ldots \ldots \ldots \ldots \ldots$

K. Rozgonyi \& S. Frey

Ensemble quasar spectral variability from the XMM-Newton Serendipitous Source

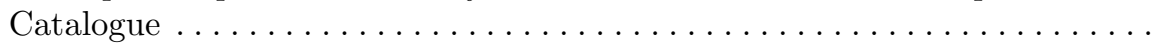

R. Serafinelli, F. Vagnetti \& R. Middei

Peculiar emission from the new VHE gamma-ray source $\mathrm{H} 1722+119$. . . . . . . .

T. Terzić, A. Stamerra, the MAGIC Coll., F. D'Ammando (for the

Fermi-LAT Coll.), C. M. Raiteri, M. Villata, F. Verrecchia \& O. Kurtanidze

Influence of self-gravity on the equilibrium structures of magnetized tori. . . . . .

A. Trova, V. Karas, P. Slaný \& J. Kovár

\section{Topic 5: Tests of fundamental theories of physics using black hole systems}

Unveiling early black holes with $J W S T \ldots \ldots \ldots \ldots \ldots \ldots \ldots \ldots \ldots \ldots \ldots \ldots \ldots$

P. Natarajan

Black holes in Einstein-Gauß-Bonnet-dilaton theory . . . . . . . . . . .

J. Luis Blázquez-Salcedo, V. Cardoso, V. Ferrari, L. Gualtieri, P. Kanti,

F. Scen Khoo, B. Kleihaus, J. Kunz, C. F. B. Macedo, S. Mojica, P. Pani

E E. Radu

Black hole superradiance as a probe of ultra-light new particles . . . . . . . .

R. Lasenby

Quantum electromagnetic phenomena far from small evaporating black holes...

S. Emelyanov

Spectroscopy of candidate electromagnetic counterparts to gravitational wave

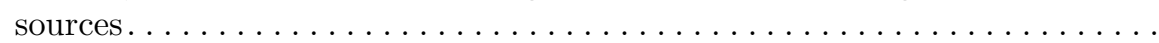

I. A. Steele, C. M. Copperwheat \& A. S. Piascik

MAGIC electromagnetic follow-up of gravitational wave alerts . . . . . . . .

B. D. Lotto, S. Ansoldi, A. Antonelli, A. Berti, A. Carosi, F. Longo \&

A. Stamerra on behalf of the MAGIC Collaboration 
On the gamma-ray burst - gravitational wave association in GW150914 . . . . .

A. Janiuk, S. Charzynski \& M. Bejger

Search for UHE neutrinos in coincidence with LIGO GW150914 event with the

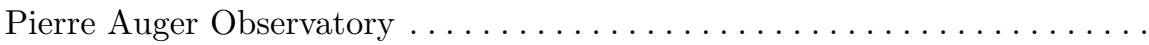

L. Yang, for the Pierre Auger Collaboration

What was the Initial Mass of Merging Black Holes in GW150914 ? . . . . . . . . .

H. Tagawa \& M. Umemura

Black holes formed by direct collapse: observational evidences . . . . . . . . .

I. F. Mirabel

\section{Topic 6: Technology Drivers and Future Capabilities}

Black hole astrophysics with HAWC, the High Altitude Water Cherenkov $\gamma$-ray

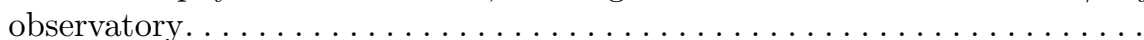

A. Carramiñana for the HAWC Collaboration

Acceleration of particles up to $\mathrm{PeV}$ energies at the galactic centre . . . . . . . .

S. Gabici, F. A. Aharonian, E. Moulin \& A. Viana

Neutrino Astronomy with IceCube . . . . . . . . . . . . . . . . . .

K. J. Meagher on behalf of the IceCube Collaboration

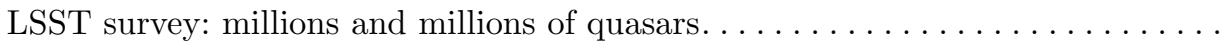

Ž. Ivezić

X-ray polarimetry - A new Window on Black Hole Systems . . . . . . . . . .

$R$. W. Goosmann on behalf of the XIPE consortium

KETJU: Post-Newtonian-Accurate Supermassive Black Hole Dynamics in GADGET-

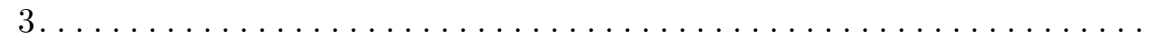

A. Rantala, P. Pihajoki \& P. H. Johansson

Ray-tracing and polarized radiative transfer in General Relativity . . . . . . . . .

P. Pihajoki, A. Rantala \& P. H. Johansson

Observing shadow of the Schwarzschild black hole in presence of a plasma ..... F. Atamurotov

Circular motion and Polish Doughnuts in NUT spacetime.

P. I. Jefremov

A solution to unconstrained Einstein's equations for a relativistic radiation sphere

L. Neslušan

MOPTOP: Multi-colour Optimised Optical Polarimeter .............

H. Jermak, I. A. Steele 6 R. J. Smith

Author index 


\section{Preface}

Black holes lie at the heart of some of the most fascinating astrophysical phenomena. The International Astronomical Union Symposium 324: "New Frontiers in Black Hole Astrophysics" marked the 100th anniversary of Schwarzschild's solution of Einstein's field equations, which set the scene for the theoretical prediction of black holes. Although they were at first considered as a purely mathematical curiosity, it is now well established that they are ubiquitously present in the Universe. In the last few decades our understanding of black holes has come an impressively long way, with the last major discovery in this field being coalescing black holes producing gravitational waves. Gravitational waves, also predicted in 1916, remained elusive for a long time - therefore, it was our outmost pleasure to mark during the symposium the first anniversary of the first direct gravitational wave detection, which started a new era in astronomy.

In addition to historical perspective, the symposium was timely also in terms of scientific and technological points of view. Technological advances across the electromagnetic spectrum and beyond to multi-messenger signature are coming of age. The new generation of gravitational wave detectors is operational, improved sensitivity in neutrino detectors is available, the multi-wavelength community has an impressive suite of ground- and space-based facilities covering a wide range of energy bands and timescales. And on the theoretical side, astrophysics communities are providing new testable predictions from advances in numerical simulations.

IAU symposium 324 was held from September 12 to 16, 2016 in Slovenia's capital city Ljubljana. It was organized by the University of Nova Gorica and opened by the Slovenian Minister of Education, Science and Sport, Dr. Maja Makovec Brenčič. The symposium brought together 130 observational and theoretical experts from 30 countries across the globe to discuss the current state-of-the-art in the astrophysics of black-hole driven systems and their exploitation in testing fundamental theories of physics. The scientific program began with a historical introduction given by Virginia Trimble and concluded with the symposium summary by Carole Mundell. The program consisted of 24 invited talks, 57 contributed talks and 30 posters. Topics of presentations and discussions spanned a wide range and included similarity and diversity of black hole systems, gamma ray bursts, tidal disruption events, active galactic nuclei, gravitational waves, black hole systems as multi-messenger sources and tools for testing theories of gravity and elementary particles, current and future large experimental facilities, and opening of new observational horizons.

Scientific highlights of the symposium included presentations and discussions on:

- Similarities and diversities of black hole systems, how a large-scale disk dynamo creates large-scale, long-lived jets; influence of time-dependent collapse history, growth of supermassive black holes and seed black holes.

- Magnetic field in black hole systems, in particular spatially resolved images of jets, polarisation, multi-messenger signals, and advances in numerical simulations of the magnetic field effects.

- Tidal Disruption Events and origin of UV/optical emission in them, rareness of relativistic events, possible unification scheme, host galaxies properties, estimation of type and mass of disrupted star, and many other puzzles unresolved due to a small number of these events discovered so far.

- The birth of gravitational wave astronomy, the information contained in gravitational wave signals (inspiral, merger and ringdown phase), potential electromagnetic signals, and possibilities of constraining the neutron star equation of state. 
- Tests of fundamental physics and black holes, including quantum gravity 'zoo' and advances in theoretical modelling of gravitational waves.

Poster's presenters were given an opportunity to give a short highlight talk of their work and to take part in the best poster competition with prizes drawn from traditional Slovenian natural produce.

Social program of the symposium included a walking tour of Ljubljana, symposium dinner and trip to Postojna Caves and Lanthieri mansion in Vipava.

Symposium program was accompanied by several events for general public. In a public lecture on Sep 14, 2016, exactly on the 1st anniversary of the first direct detection of gravitational waves, Sheila Rowan presented a captivating story about detection of gravitational waves, in front of the packed full symposium hall. Exhibitions about black holes were on view during the symposium week in the National Assembly of the Republic of Slovenia and in the Cankar Centre, the symposium venue. After the symposium, one exhibition was put on display in Slovenian Museum of Natural History, while the other one is travelling and visiting Slovenian schools. To maximize educational outreach we invited all interested teachers to the symposium, to view the posters and listen to review talks. We organized a special teachers workshop on black holes in collaboration with the Society of Mathematicians, Physicists and Astronomers of Slovenia. General public and media exhibited large interest in the symposium, with all major TV stations and journals reporting news about the symposium and/or publishing interviews with symposium participants.

We were indeed very happy and honoured to host this symposium! It was not only the first International Astronomical Union Symposium held in Slovenia, but also the largest professional astronomy meeting in Slovenia so far.

I hope that these proceedings will serve as a summary of the highly interesting contributions and fruitful discussions held during the symposium, and that they will be especially useful to researchers and graduate students engaged in many different, yet all very exciting, research fields related to black holes.

Andreja Gomboc

Chair of the $S O C$ and $L O C$

Ajdovščina, March 8, 2017 


\section{Acknowledgements}

The symposium was sponsored by travel grants from the International Astronomical Union and supported by the International Astronomical Union Divisions A (Fundamental Astronomy), B (Facilities, Technologies and Data Science), C (Education, Outreach and Heritage), D (High Energy Phenomena and Fundamental Physics), J (Galaxies and Cosmology), and Commission 44 (Space \& High Energy Astrophysics).

We are grateful to members of the Scientific Organizing Committee and the Local Organizing Committee for their highly valuable contributions.

Many special thanks to Carole Mundell for co-chairing the symposium!

Financial support by Aresis Ltd. and IPZ d.o.o. is gratefully acknowledged. 
Scientific Organizing Committee:

Andreja Gomboc, University of Nova Gorica (chair)

Carole Mundell, University of Bath (co-chair)

Felix Aharonian, Max Planck Institute for Nuclear Physics

Dave Burrows, The Pennsylvania State University

Paula Chadwick, Durham University

Stefano Covino, INAF- Astronomical Observatory Brera - Merate

Gabriela Gonzales, Louisiana State University

Shiho Kobayashi, Liverpool John Moores University

Stefanie Komossa, Max Planck Institute for Radio Astronomy

Elina Lindfors, University of Turku

Stephan Rosswog, Stockholm University

Subir Sarkar, University of Oxford

Kenji Toma, Tohoku University

Diana Worrall, University of Bristol

\section{Local Organizing Committee:}

Andreja Gomboc (chair)

Aurora Clerici

Uroš Kostić

Gašper Kukec Mezek

Marta Trini

Gabrijela Zaharijaš

Lili Yang

Sponsors:

Aresis Ltd.

IPZ d.o.o.

Cover image text:

Logo of the IAU Symposium 324 on the background of NGC 6240 (Logo Credit: B. Žalar, Photo Credits: X-ray: NASA/CXC/MIT/C. Canizares, M. Nowak; Optical: NASA/STScI) 
CONFERENCE PHOTOGRAPH

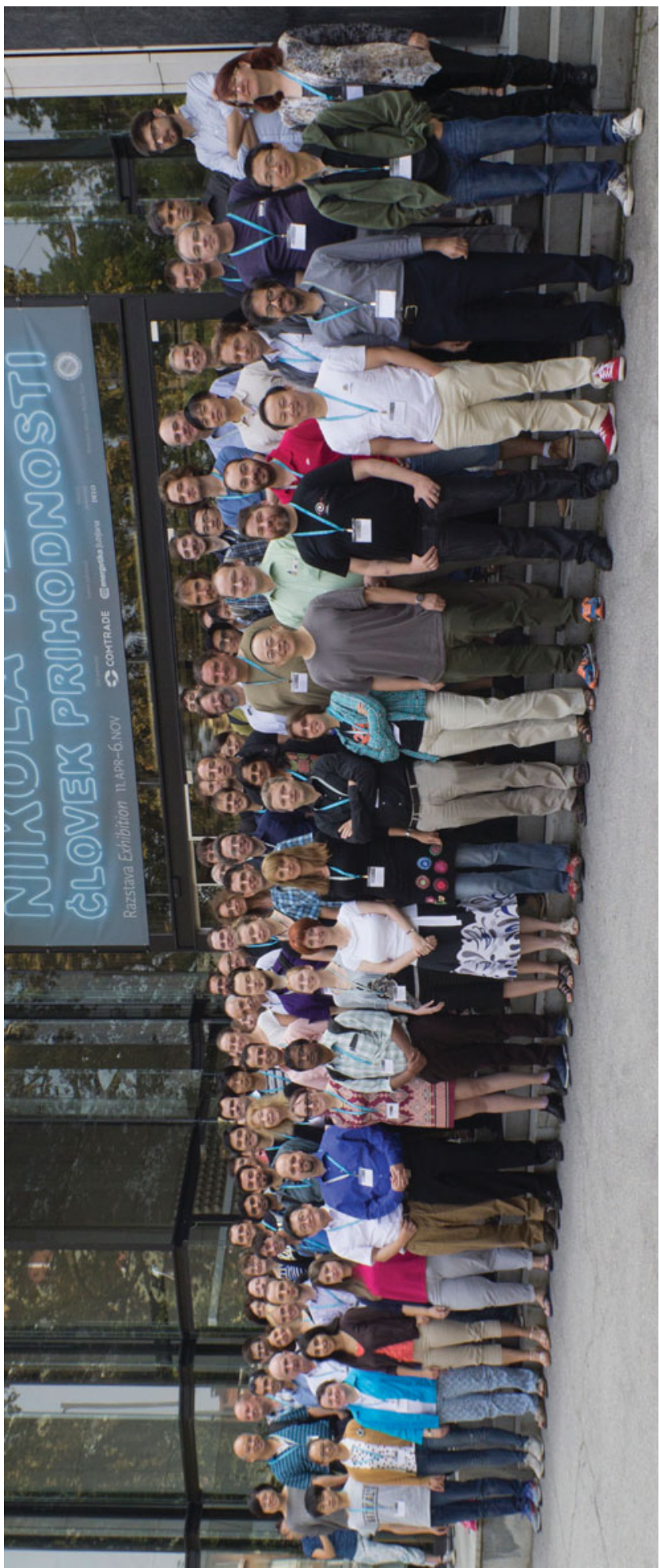




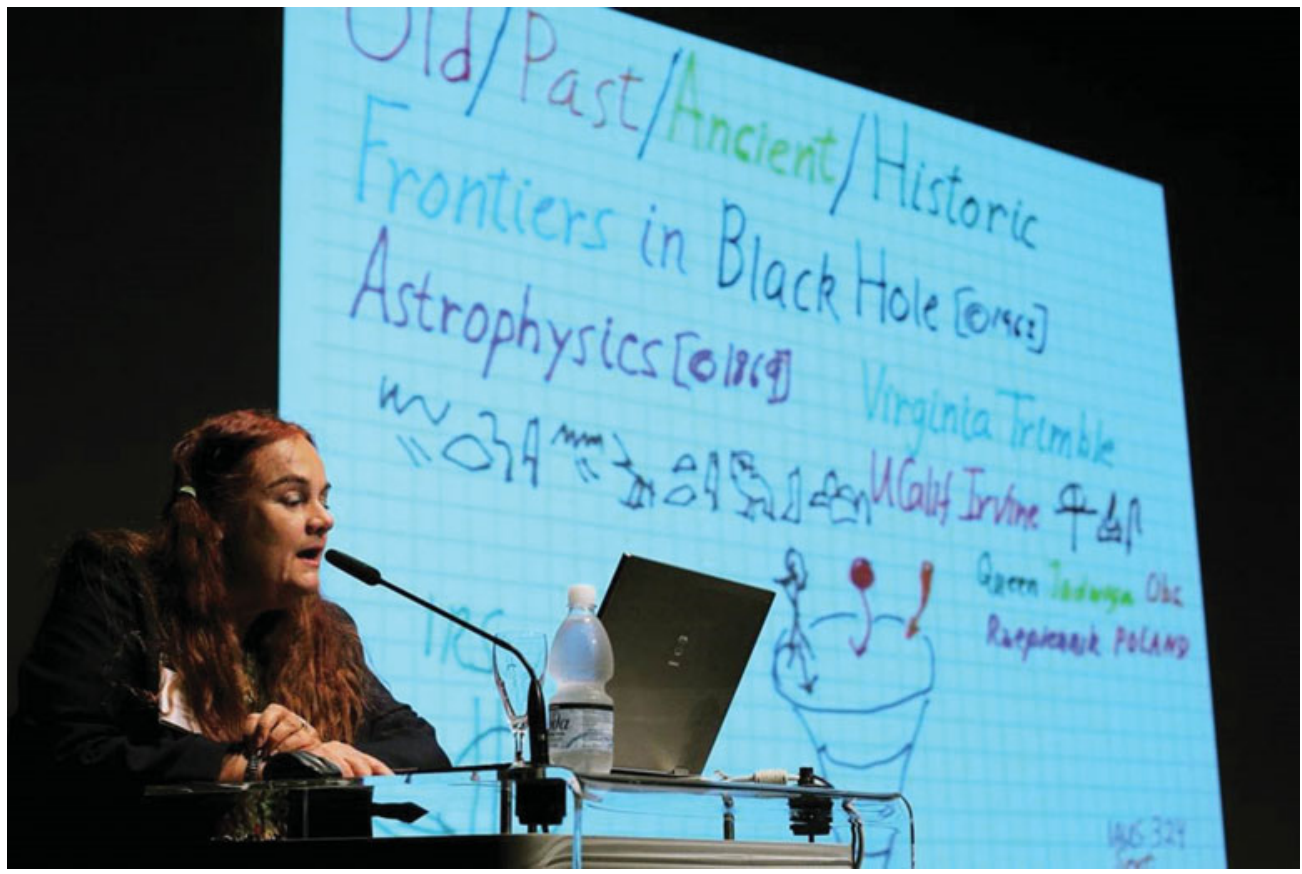

Photo 1. Introductory review of the black hole concept and development of black hole research field given by Prof. Virginia Trimble (12 Sep 2016, photo: D. Novakovič/STA).

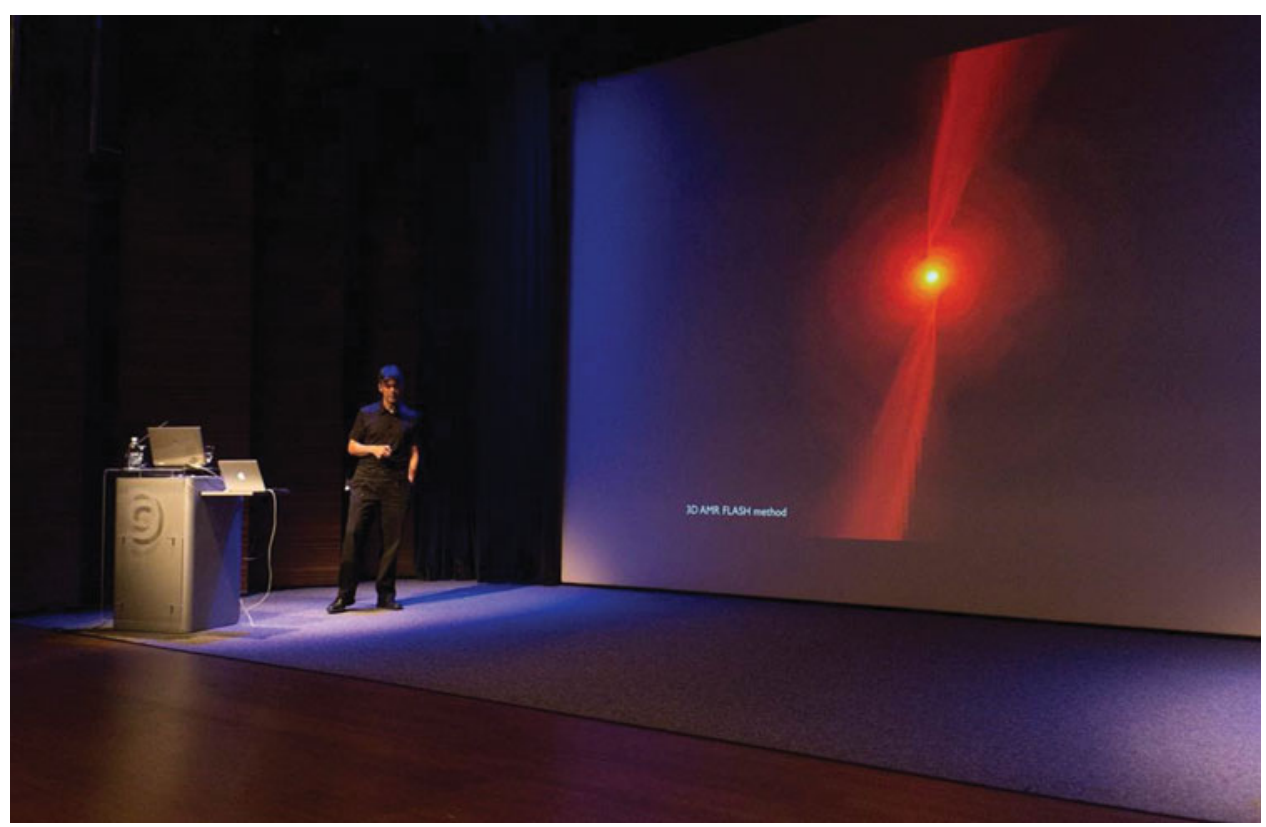

Photo 2. Enrico Ramirez-Ruiz reviewing numerical simulations of tidal disruption events (13 Sep 2016, photo: G. Kukec Mezek). 


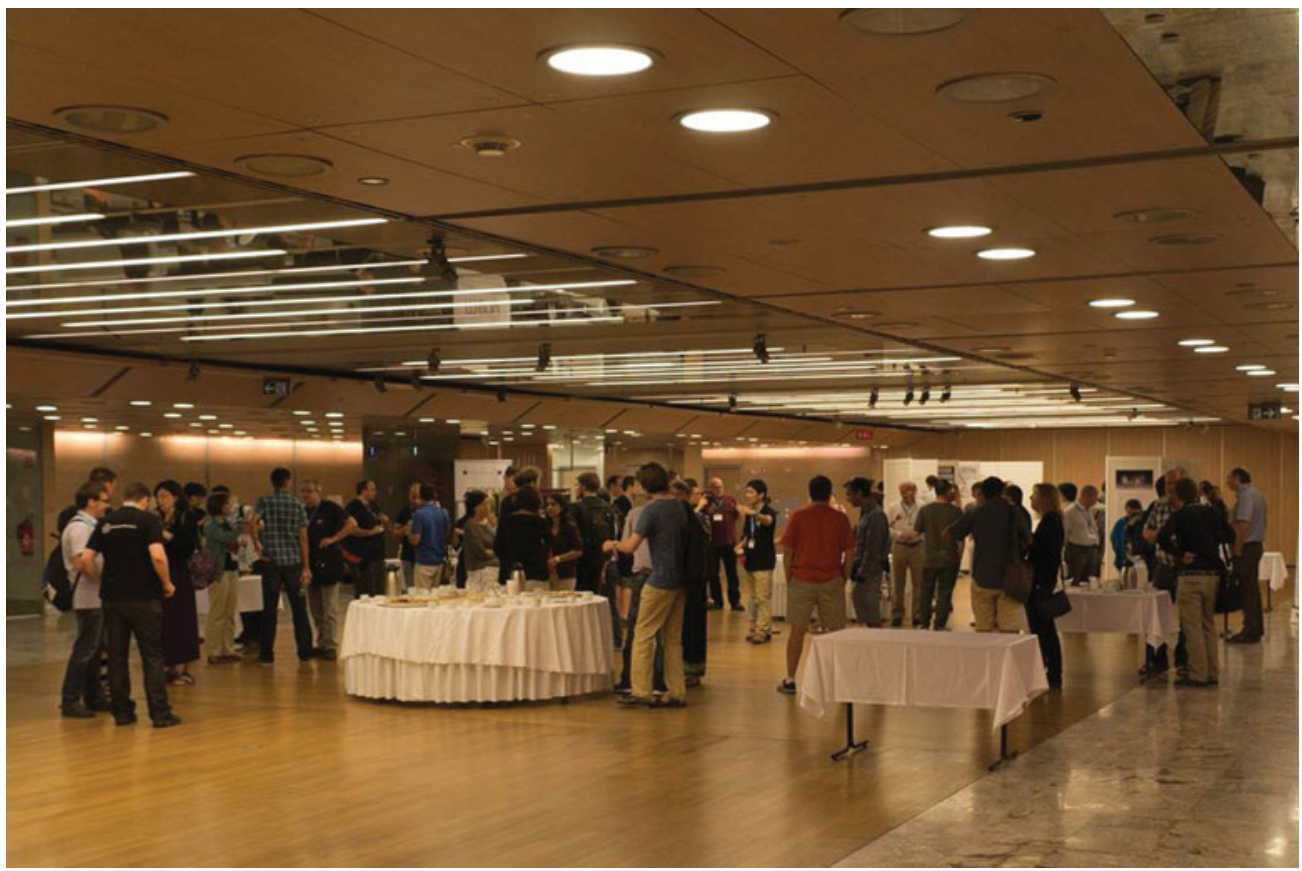

Photo 3. Discussions during a coffee break (photo: G. Kukec Mezek).

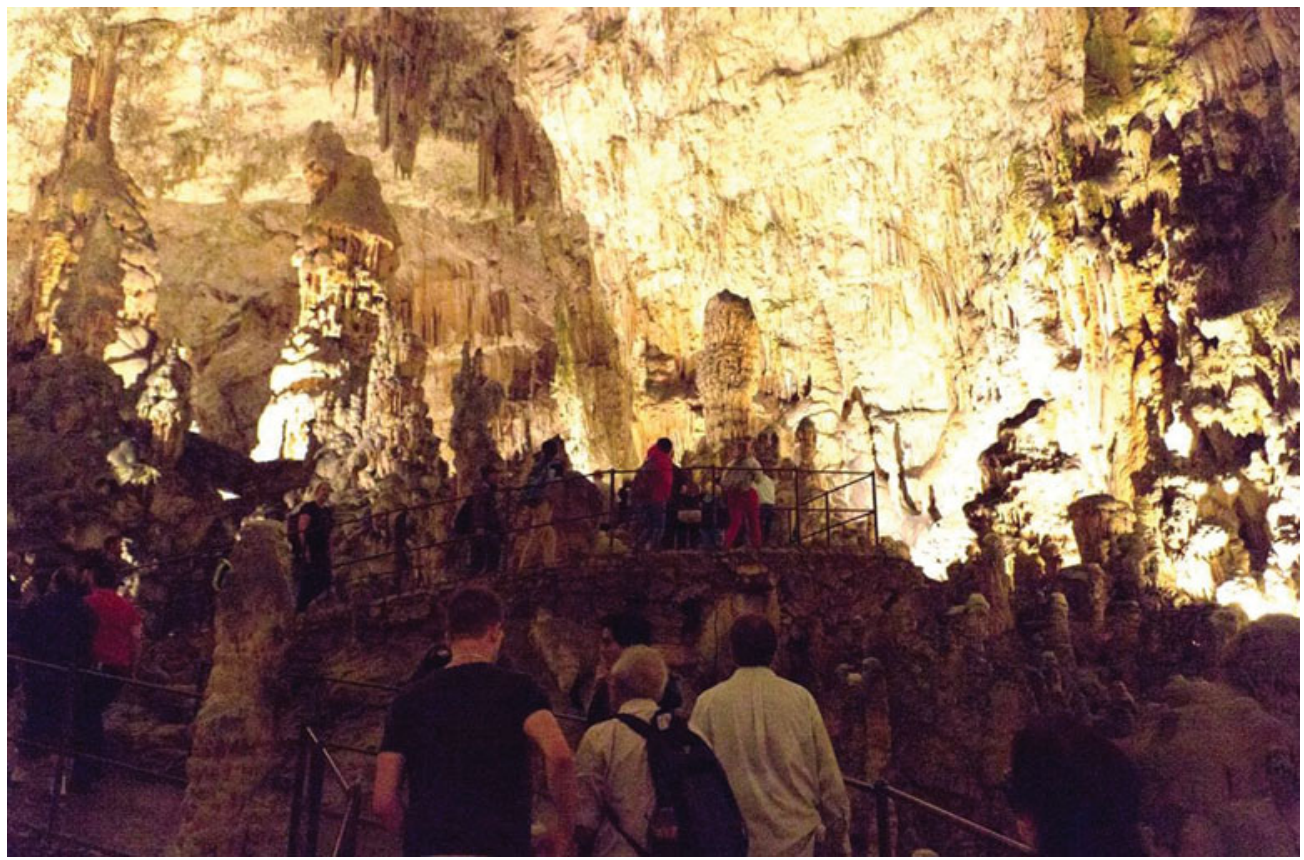

Photo 4. Symposium trip to Postojna Cave (14 Sep 2016, photo: G. Kukec Mezek). 


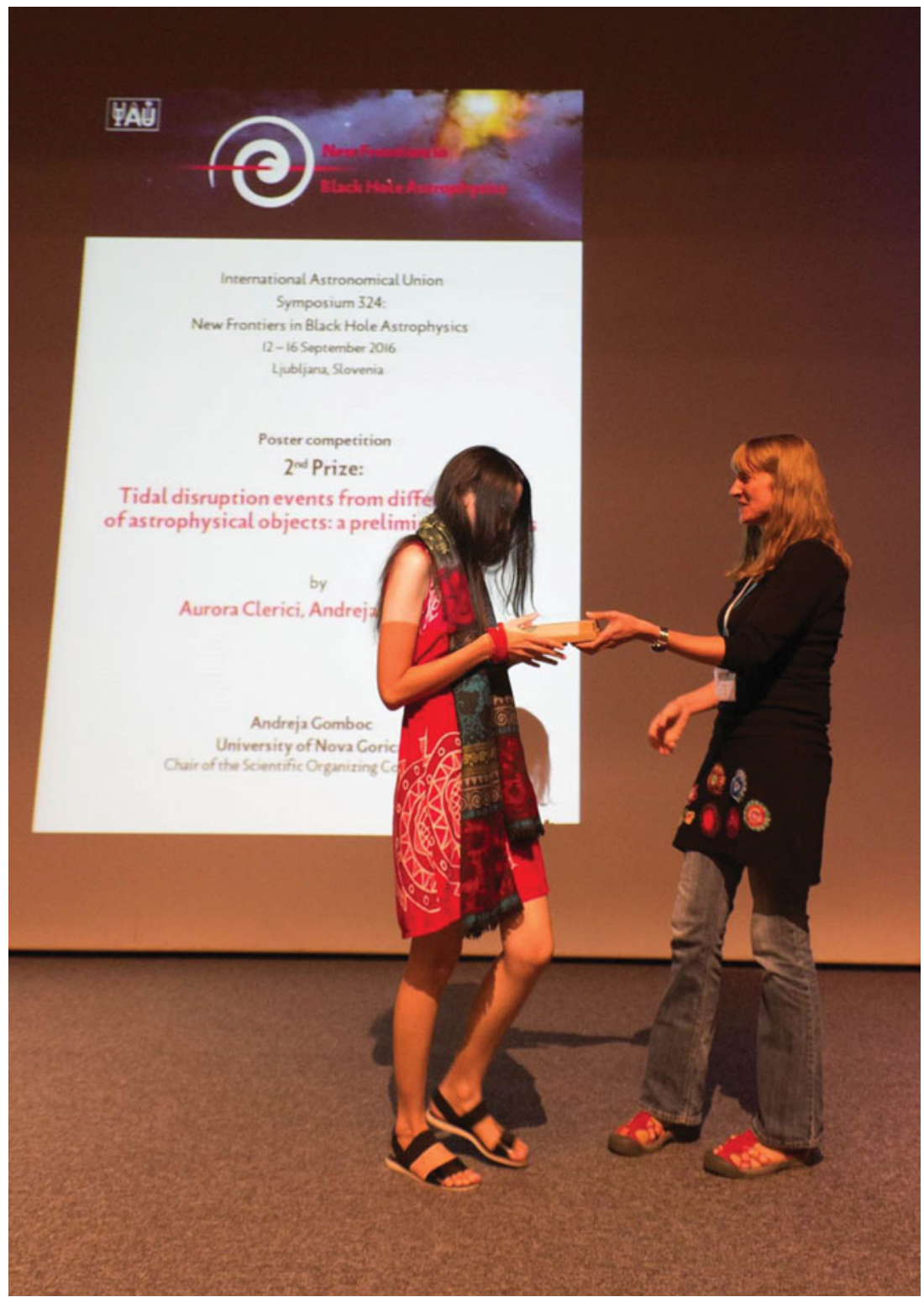

Photo 5. Announcement of the poster competition winners (15 Sep 2016, photo: G. Kukec Mezek). 


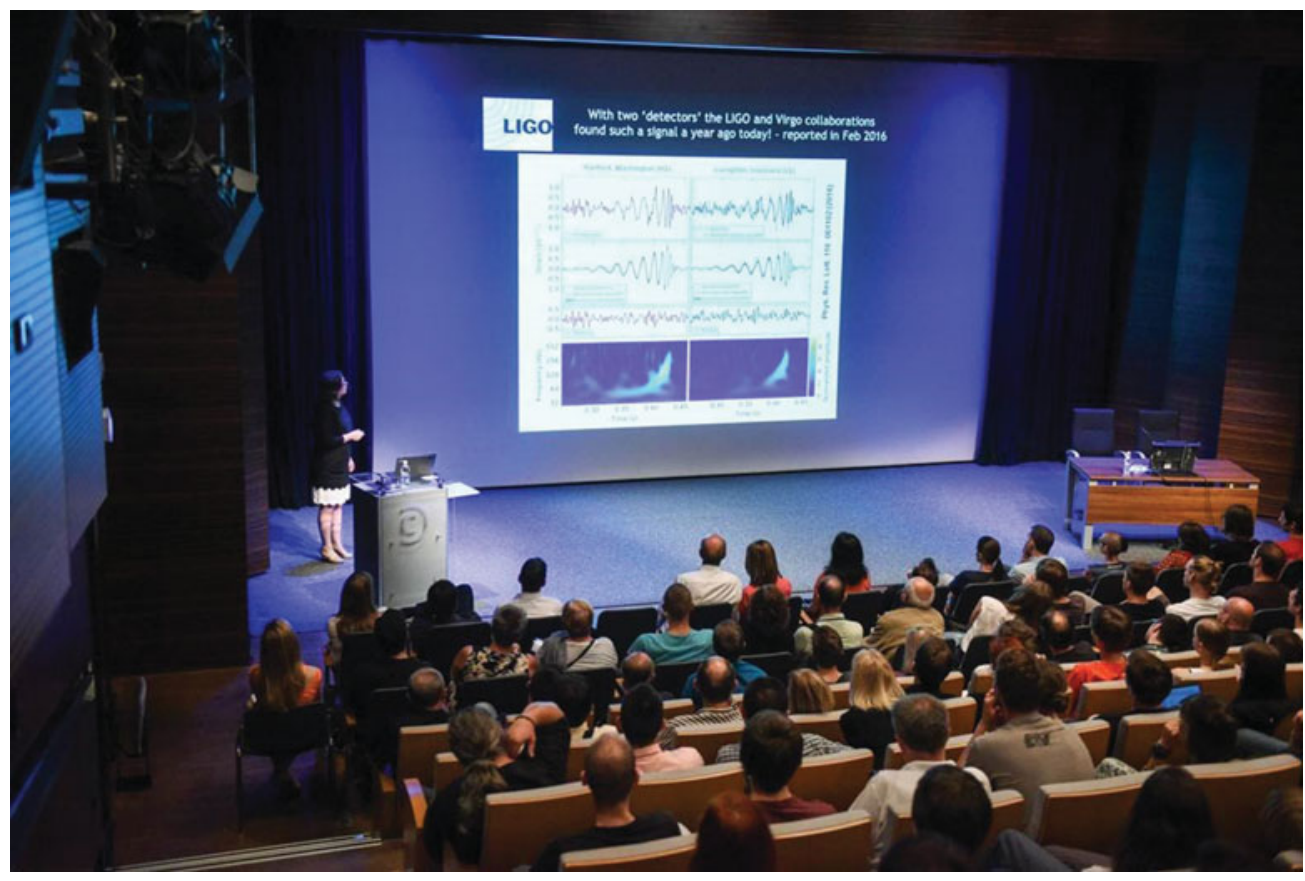

Photo 6. Public lecture on gravitational waves given by Sheila Rowan on the first anniversary of the first direct detection by the LIGO observatory (14 Sep 2016, photo: N. Tejić/STA).

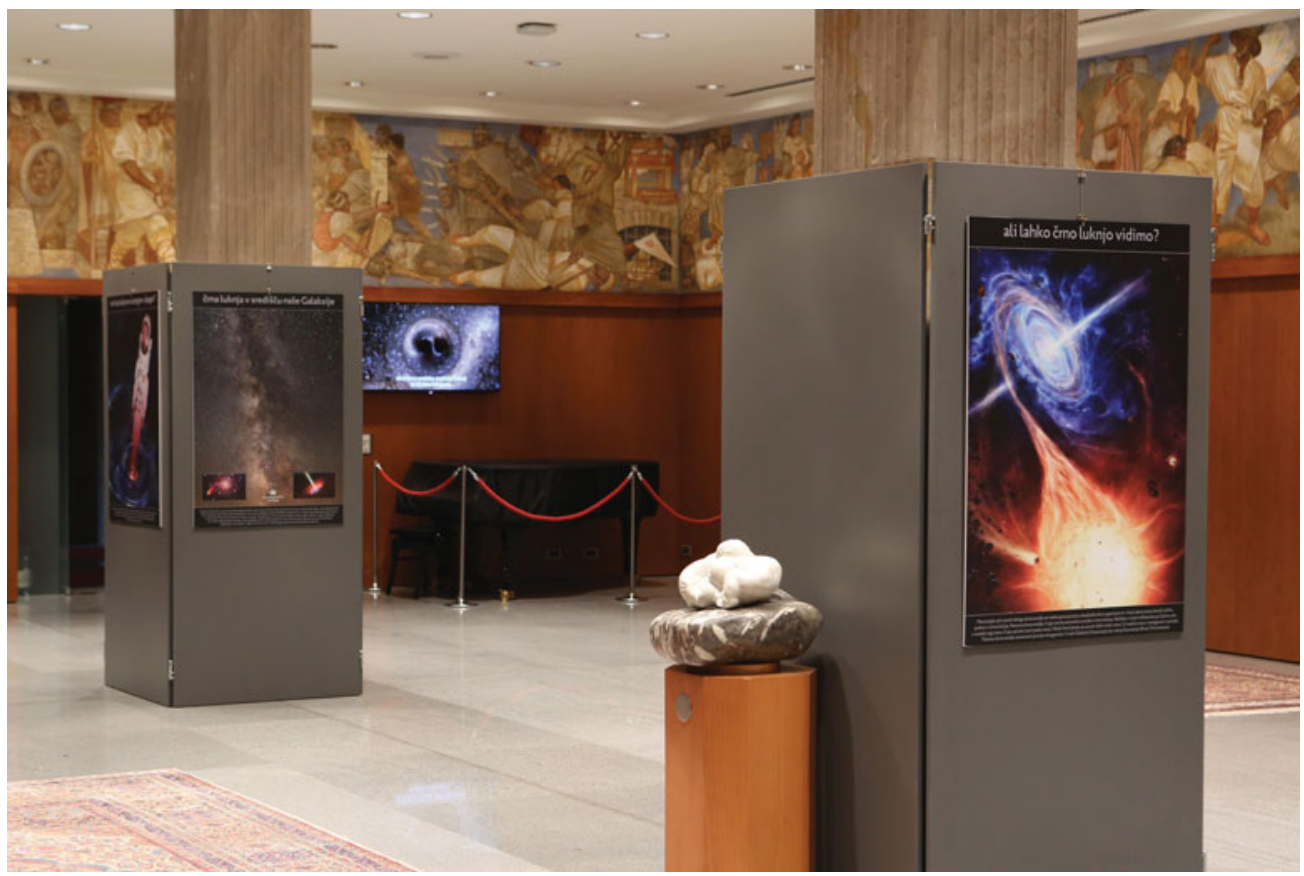

Photo 7. Exhibition about black holes in the National Assembly of the Republic of Slovenia (12-16 Sep 2016, photo: B. Peršolja). 


\section{PARTICIPANTS}

\author{
Tal Alexander \\ Cornelia Arcaro
}

Farruh Atamurotov

Patrycja Bagińska

Charles Bailyn

Maxim Barkov

Josefa Becerra Gonzalez

Alessio Berti

Geoffrey Bicknell

Akos Bogdan

Edi Bon

Nataša Bon

Iztok Bončina

Željka Marija Bošnjak

Martin Bourne

Katja Bricman

Alberto Carraminana

Brad Cenko

Paula Chadwick

Sunil Chandra

Marisa Charisi

Surajit Chattopadhyay

Aurora Clerici

Carlos Coimbra Araujo

Stefano Covino

Andrej Čadež

Miha Černetič

Colin Degraf

Barbara De Lotto

Anja Dobravec

Dijana Dominis Prester

Ioana Duţan

Viacheslav Emelyanov

Dimitrios Emmanoulopoulos

Dunja Fabjan
Weizmann Inst. of Science,

Israel

INFN Padova \& Padova Univ., cornelia.arcaro@pd.infn.it Italy

Ulugh Beg Astronomical Institute, Uzbekistan

Adam Mickiewicz Univ., Poland patibag@gmail.com

Yale Univ. / Yale-NUS College, charles.bailyn@yale.edu USA

University of Potsdam, Germany

NASA Goddard SFC, USA jbecerragonzalez@gmail.com

INFN, University of Trieste, alessioberti90@gmail.com Italy

RSAA, Australian National geoff.bicknell@anu.edu.au Univ., Australia

Harvard-Smithsonian CfA, USA abogdan@cfa.harvard.edu

Astronomical Observatory, Serbia

Astronomical Observatory, Serbia

Astronomsko društvo Javornik, iztok.boncina@guest.arnes.si Slovenia

University of Rijeka, Croatiarbosnjak@uniri.hr

Kavli Institute for Cosmology, IoA, UK

University of Ljubljana, Slovenia

INAOE, Mexico

NASA GSFC, USA

University of Durham, UK

TIFR, India

Columbia University, USA

PCMT, India

University of Nova Gorica, Slovenia

Univ. Federal do Paraná, Brazil

INAF / Brera, Italy

University of Ljubljana, Slovenia

University of Ljubljana, Slovenia

IoA, University of Cambridge, UK

University of Udine \& INFN, Italy

University of Ljubljana, Slovenia

University of Rijeka, Croatia

Institute of Space Science, Romania

KIoT, IoTP, Germany viacheslav.emelyanov@kit.edu

University of Southampton, UK d.emmanoulopoulos@soton.ac.uk

University of Ljubljana, dunja.fabjan@fmf.uni-lj.si 
Vandad Fallah Ramazani

Juan Antonio Fernández

Ontiveros

Sandor Frey

Krisztina Gabányi

Stefano Gabici

Mikhail Garasev

Sarah Gibson

Andreja Gomboc

Rene Goosmann

Diego Götz

Minfeng $\mathbf{G u}$

Mark Hannam

Carlos Herdeiro

Talvikki Hovatta

Xiaoli Huang

Samo Ilc

Susumu Inoue

Kunihito Ioka

Željko Ivezić

Agnieszka Janiuk

Taj Jankovič

Pavel Jefremov

Helen Jermak

Aleksej Jurca

Anton Jurca Vassilis

Karamanavis

Sergey Karpov

Shai Kaspi

Nobuyuki Kawai

Norita Kawanaka

Minjin Kim

Shiho Kobayashi

Serguei Komissarov

Stefanie Komossa

Uroš Kostić

Gašper Kukec Mezek

Pawan Kumar

Jutta Kunz

Samo Kupper

Gavin Lamb

Robert Lasenby
University of Turku, Finland

Inst. de Astrof. de Canarias, Spain

Satellite Geod. Observatory, Hungary

Satellite Geod. Observatory, Hungary

Laboratoire APC, France

Institute of Applied Physics, Russian Federation

University of Leicester, UK

University of Nova Gorica, Slovenia

Obs. astron. de Strasbourg, France

CEA Saclay, France

Shanghai Astronomical Obs., CAS, China

Cardiff University, UK

Aveiro University, Portugal

Aalto Univ. Metsahovi Radio Obs., Finland

Guangxi University, China

University of Ljubljana, Slovenia

RIKEN, Japan

YITP, Kyoto University, Japan

University of Washington, USA

Center for Theor. Physics, PAS, Poland

University of Ljubljana, Slovenia

ZARM, Univ. Bremen, Germany

Lancaster University, UK

Gimnazija Bežigrad, Slovenia

Max-Planck-Inst. für Radioastr., Slovenia Germany

SAO RAS, Russian Federation

Tel Aviv University, Israel

Tokyo Inst. of Technology, Japan

University of Tokyo, Japan

Korea Astr. and Space Sc. Inst., mkim@kasi.re.kr Republic of Korea

ARI, Liverpool JMU, UK

University of Leeds, UK

MPIfR, Germany

Aalta Lab d.o.o., Slovenia

University of Nova Gorica, Slovenia

Univ. of Texas at Austin, USA

University of Oldenburg, Germany

Fizikalno društvo Kočuha, Austria

ARI, Livrepool JMU, UK

Perimeter Institute, Canada vafara@utu.fi

jafo@iac.es

frey.sandor@fomi.hu

gabanyik@sgo.fomi.hu

gabici@apc.in2p3.fr

garasev@appl.sci-nnov.ru

slg44@le.ac.uk

andreja.gomboc@ung.si

rene.goosmann@astro.unistra.fr

diego.gotz@cea.fr

gumf@shao.ac.cn

mark.hannam@astro.cf.ac.uk

herdeiro@ua.pt

talvikki.hovatta@aalto.fi

1536756072@qq.com

samo.ilc37@gmail.com

susumu.inoue@riken.jp

kunihito.ioka@yukawa.kyotou.ac.jp

ivezic@astro.washington.edu

agnes@cft.edu.pl

taj.jankovic@gmail.com

paulefremov@gmail.com

h.jermak@lancaster.ac.uk

vkaraman@mpifr-

bonn.mpg.de

karpov.sv@gmail.com

shai@wise.tau.ac.il

nkawai@phys.titech.ac.jp

norita@astron.s.u-

tokyo.ac.jp

S.Kobayashi@ljmu.ac.uk s.s.komissarov@leeds.ac.uk stefanie.komossa@gmx.de uros.kostic@aalta-lab.com gasper.kukec@ung.si

pk@astro.as.utexas.edu

jutta.kunz@uni-

oldenburg.de

samo.kupper@expi.at

g.p.lamb@2010.ljmu.ac.uk robertlasenby@gmail.com 
Shuangliang Li

Enwei Liang

Elina Lindfors

Tamilan Mageshwaran

Pablo Marchant

Paola Marziani

Jin Matsumoto

Tatsuya Matsumoto

Kevin Meagher

Attila Meszaros

Felix Mirabel

Carole Mundell

Saeede Nafooshe

Priyamvada Natarajan

Lubos Neslusan

Zsolt Paragi

Qiuhe Peng

Bradley Peterson

Pauli Pihajoki

Peter Raffai

Enrico Ramirez-Ruiz

Antti Rantala

Sheila Rowan

Richard Saxton

Luka Seliškar

Roberto Serafinelli

Samo Stanič

Iain Steele

Lisa K. Steinborn

Petra Sukova

Tachibana Yutaro

Hiromichi Tagawa

Ataru Tanikawa

Nial Tanvir

Alexander Tchekhovskoy

Tomislav Terzić

Kenji Toma

Virginia Trimble

\section{Marta Trini}

Audrey Trova

Rok Vogrinčič

Serguei Vorobiov
Shanghai Astronomical Obs., China

Guangxi University, China

Tuorla Obs., Univ. of Turku, Finland

Indian Institute of Astrophysics, India

Universität Bonn, Germany

INAF - Astron. Obs. Padova, Italy

RIKEN, Japan

Kyoto University, Japan

Univ. libre de Bruxelles, Belgium

Charles University, Czech Republic

CEA-Saclay-France, France

University of Bath, UK

Trieste University, Italy

Yale University, USA

AISAS, Slovakia

Joint Inst. for VLBI ERIC, Netherlands

Nanjing University, China

Ohio State Univ./STSI, USA

University of Helsinki, Finland

Lorand Eotvos University, Hungary

UCSC, USA

University of Helsinki, Finland

University of Glasgow, UK

XMM SOC / ESAC, Spain

University of Ljubljana, Slovenia

Univ. of Rome 'Tor Vergata', Italy

University of Nova Gorica, Slovenia

ARI, Liverpool JMU, UK

Univ.-Sternwarte München, Germany

CTP PAS, Poland

Tokyo Inst. of Technology, Japan

University of Tokyo, Japan

University of Tokyo, Japan

University of Leicester, UK

UC Berkeley, USA

University of Rijeka, Croatia

Tohoku University, Japan

UC Irvine \& Queen Jadwiga Obs., USA

University of Nova Gorica, Slovenia

AIASCR, Czech Republic

University of Ljubljana, Slovenia

University of Nova Gorica, Slovenia lisl@shao.ac.cn

\section{LEW@GXU.EDU.CN}

elilin@utu.fi

mageshwaran@iiap.res.in

pamarca@gmail.com

paola.marziani@oapd.inaf.it

jin.matsumoto@riken.jp

matsumoto@tap.scphys.kyoto-

u.ac.jp

kmeagher@ulb.ac.be

meszaros@cesnet.cz

felix.mirabel@cea.fr c.g.mundell@bath.ac.uk saeede.nafooshe@ung.si priyamvada.natarajan@yale.edu ne@ta3.sk

zparagi@jive.eu

qhpeng@nju.edu.cn peterson.12@osu.edu pauli.pihajoki@helsinki.fi praffai@bolyai.elte.hu

enrico@ucolick.org antti.rantala@helsinki.fi sheila.rowan@glasgow.ac.uk rsaxton@sciops.esa.int lmdogg@gmail.com

roberto.serafinelli@roma2.infn.it

samo.stanic@ung.si

i.a.steele@ljmu.ac.uk steinborn@usm.lmu.de

psukova@cft.edu.pl tachibana@hp.phys.titech.ac.jp

tagawahr@nao.ac.jp

tanikawa@ea.c.u-

tokyo.ac.jp

nrt3@le.ac.uk

atchekho@berkeley.edu

tomislav.terzic@gmail.com

toma@astr.tohoku.ac.jp

vtrimble@astro.umd.edu

marta.trini@ung.si

trova@asu.cas.cz

rokvogrincic@gmail.com

sergey.vorobyev@ung.si 
Amri Wandel

Vojtěch Witzany

Lukasz Wyrzykowski

Lili Yang

Farhad Yusef-Zadeh

Gabrijela Zaharijas

Luca Zampieri

Sebastjan Zamuda

Danilo Zavrtanik

Shuqing Zhong

Tomaž Zwitter

Maruša Žerjal
Hebrew Univ. of Jerusalem, Israel

ZARM, Univ. Bremen, Germany

Warsaw Universit, Poland

University of Nova Gorica, Slovenia

Northwestern University, USA

University of Nova Gorica, Slovenia

INAF-Ast. Obs. Padova, Italy

Gimnazija Bežigrad, Slovenia

University of Nova Gorica, Slovenia

Guangxi University, China

University of Ljubljana, Slovenia

University of Ljubljana, Slovenia amri@huji.ac.il

witzany@zarm.uni-

bremen.de

lw@astrouw.edu.pl

lili.yang@ung.si

zadeh@northwestern.edu gabrijela.zaharijas@ung.si

luca.zampieri@oapd.inaf.it

danilo.zavrtanik@ung.si

$1548771042 @ q q . c o m$

tomaz.zwitter@fmf.uni-lj.si

marusa.zerjal@fmf.uni-lj.si 